\title{
MODULES WHOSE ENDOMORPHISM RINGS HAVE ISOMORPHIC MAXIMAL LEFT AND RIGHT QUOTIENT RINGS
}

\author{
SOUMAYA MAKDISSI KHURI
}

\begin{abstract}
Let ${ }_{R} M$ be a left $R$-module such that $\operatorname{Hom}_{R}(M, U) \neq 0$ for any nonzero submodule $U$ of $M$, let $E(M)$ denote the injective hull of $M$, and let $B$ (resp. $A$ ) denote the ring of $R$-endomorphisms of $M$ (resp. $E(M)$ ). It is known that if $M$ is nonsingular then $B$ is left nonsingular and $A$ is the maximal left quotient ring of $B$. We give here necessary and sufficient conditions on $M$ for $B$ to be right nonsingular and for $A$ to be the maximal right quotient ring of B.
\end{abstract}

1. Introduction and preliminaries. In [5], Utumi gave the solution of the following problem: given a ring $S$ which is both left and right nonsingular, when is the maximal left quotient ring (MLQR) of $S$ isomorphic to its maximal right quotient ring (MRQR)? He proved that this holds if and only if the converses of the nonsingular properties hold in $S$, namely, if and only if every left ideal of $S$, which has zero right annihilator, is essential in $S$ and every right ideal of $S$, which has zero left annihilator, is essential in $S$ [5, Theorem 3.3]. We consider here analogous questions for the endomorphism ring of an $R$-module.

Let ${ }_{R} M$ be a left $R$-module, where $R$ is a ring with 1 , and $B=\operatorname{End}_{R}(M)$ its ring of $R$-endomorphisms. The following notation will be used in the sequel: If $U$ is a submodule of $M$, then

$$
\begin{gathered}
I_{B}(U)=\{b \in B: M b \subset U\}, \quad r_{B}(U)=\{b \in B: U b=0\}, \\
l_{R}(U)=\{r \in R: r U=0\} .
\end{gathered}
$$

If $J$ is a right ideal of $B$, then $l_{M}(J)=\{m \in M: m J=0\} . X \subset^{\prime} Y$ means that $X$ is an essential submodule of $Y$, i.e. $X$ intersects nontrivially every nonzero submodule of $Y$; in case $I$ is a left, right or two-sided ideal of a ring $S$, then ${ }_{s} I C^{\prime}$ ${ }_{s} S$ (resp. $I_{s} \subset^{\prime} S_{s}$ ) will indicate that $I$ is essential in $S$ as a left (resp. right) ideal of $S$.

Recalling that ${ }_{R} M$ is said to be nonsingular in case the only submodule of $M$ with essential (left) annihilator in $R$ is the zero submodule-in our notation: $l_{R}(U) \subset^{\prime}{ }_{R} R \Rightarrow U=0$-we will call $M$ cononsingular in case the only submodule of $M$ with essential (right) annihilator in $B$ is the zero submodule-i.e. $r_{B}(U) C^{\prime}$ $B_{B} \Rightarrow U=0$. Let $E(M)$ denote the injective hull of $M$ and $A=\operatorname{End}_{R}[E(M)]$ its ring of $R$-endomorphisms. It is known that if $M$ is nonsingular then $A$ is a (von Neumann) regular left, self-injective ring. If we impose a mild nondegeneracy

Received by the editors June 23, 1981.

1980 Mathematics Subject Classification. Primary 16A08, 16A65.

$K e y$ words and phrases. Endomorphism rings, nonsingular modules and rings, maximal quotient rings, Utumi rings. 
condition on $M$, namely assuming $M$ is retractable, i.e. $I_{B}(U) \neq 0$ for any nonzero submodule $U$ of $M$, then, when ${ }_{R} M$ is nonsingular, $B$ is left nonsingular and $A$ is the MLQR of $B$ [4]. It is natural to ask here: what properties of $M$ will make $B$ also right nonsingular and what properties of $M$ will make $A$ isomorphic to the MRQR of $B$ ?

In answer to the first question, we show in Proposition 1 that, for a retractable nonsingular ${ }_{R} M, B$ is right nonsingular if and only if $M$ is cononsingular. As for the second question, it turns out that the required conditions on $M$ closely parallel the conditions of Utumi mentioned above; specifically, we show in Theorem 2 that $B$ has isomorphic MLQR and MRQR if and only if

(a) any (left) submodule of $M$ with zero (right) annihilator in $B$ is essential in $M$-i.e. $r_{B}(U)=0 \Rightarrow U \subset^{\prime} M$; and

(b) any right ideal of $B$ with zero (left) annihilator in $M$ is essential in $B$-i.e. $l_{M}(J)=0 \Rightarrow J_{B} \subset^{\prime} B_{B}$.

We note that property (a) is the converse of the following well-known property of a nonsingular module: if ${ }_{R} M$ is nonsingular then any essential submodule of $M$ has zero annihilator in $B$-i.e.: $U \subset^{\prime} M \Rightarrow r_{B}(U)=0$; while property (b) is the converse of a corresponding property of cononsingular modules: if $M$ is cononsingular then any essential right ideal of $B$ has zero kernel-i.e. $J_{B} \subset^{\prime} B_{B} \Rightarrow$ $l_{M}(J)=0$. Examples of modules satisfying the various conditions are given in the last paragraph.

2. Endomorphism rings with isomorphic left and right quotient rings. Henceforth, unless otherwise indicated, let ${ }_{R} M$ be a retractable, nonsingular left $R$ module, so that, in particular, $B$ is left nonsingular and $A$ is the MLQR of $B$.

PROPOSITION 1. $B$ is right nonsingular if and only if $M$ is cononsingular.

Proof. Assume that $B$ is right nonsingular, so that any element of $B$ with essential right annihilator must be zero. If $U$ is any submodule of $M$, then clearly $I_{B}(U) r_{B}(U)=0$. Hence, given the right nonsingularity of $B$, if $r_{B}(U)$ is essential in $B$, then $I_{B}(U)=0$, which implies, by retractability of $M$, that $U=0$. Therefore $M$ is cononsingular.

Conversely, assume that $M$ is cononsingular, and suppose that $b J=0$ for some $b$ in $B$, with $J$ an essential right ideal of $B$. Then $M b J=0$ implies that $M b$ is contained in $l_{M}(J)$; hence $r_{B}(M b)$ contains $r_{B} l_{M}(J)$ which contains $J$. Since $J$ is essential in $B$, this implies that $r_{B}(M b)$ is essential in $B$, hence, by cononsingularity of $M, M b=0$, i.e. $b=0$ and $B$ is right nonsingular.

A left nonsingular ring $S$, i.e. one in which every essential left ideal has zero right annihilator, is called a left Utumi ring in case any left ideal of $S$ with zero right annihilator in $S$ is essential in $S$. In a nonsingular left $R$-module ${ }_{R} M$, an essential (left) submodule, $U$, has zero (right) annihilator in $B-U \subset^{\prime} M \Rightarrow r_{B}(U)=0$. We will call a nonsingular ${ }_{R} M$ a Utumi module in case the converse of this property holds in $M$, i.e. in case any submodule of $M$ with zero annihilator in $B$ is essential in $M-r_{B}(U)=0 \Rightarrow U C^{\prime} M$.

The definition of a right Utumi ring is the right-left symmetry of the definition of a left Utumi ring. Using this terminology, Utumi's theorem may be restated as follows: A right and left nonsingular ring $S$ has isomorphic MLQR and MRQR if and only if $S$ is both right and left Utumi. If ${ }_{R} M$ is cononsingular, it follows easily 
that any essential right ideal of $B$ has zero kernel in $M-J_{B} \subset^{\prime} B_{B} \Rightarrow l_{M}(J)=0$; let us call a cononsingular ${ }_{R} M$ a co-Utumi module in case the converse of this property holds in $M$, i.e. in case any right ideal of $B$ with zero annihilator in $M$ is essential in $B-l_{M}(J)=0 \Rightarrow J_{B} \subset^{\prime} B_{B}$.

Our main result may now be stated as follows:

THEOREM 2. If $M$ is a retractable, nonsingular, cononsingular left $R$-module, then $A=\operatorname{End}_{R}(E(M))$ is both the $M L Q R$ and the $M R Q R$ of $B=\operatorname{End}_{R}(M)$ if and only if $M$ is both a Utumi and a co-Utumi module.

Theorem 2 follows immediately from the following two lemmas.

LEMMA 3. $B$ is a left Utumi ring if and only if $M$ is a Utumi module.

Proof. It is shown in [5] that the left nonsingular ring $B$ is a left Utumi ring if and only if $B$ has nonzero intersection with every nonzero right ideal of its MLQR, $A$ [5, Theorem 2.2]. But, by Theorem 3.5 of [3] ((iv) $\Rightarrow\left(\right.$ iii)), since $A=\operatorname{End}_{R}(E(M))$, this holds if and only if $r_{B}(U)=0$ for every $U$ which is not essential in $M$, i.e. if and only if $M$ is a Utumi module.

LEMMA 4. If $M$ is cononsingular, then $B$ is a right Utumi ring if and only if $M$ is a co-Utumi module.

Proof. Assume that $B$ is right Utumi and suppose that $l_{M}(J)=0$ for some right ideal, $J$, of $B$. Then $I_{B} l_{M}(J)=0$. But $I_{B} l_{M}(J)$ is equal to the left annihilator, $\mathcal{L}(J)$, of $J$ in $B$. Hence, the right Utumi property implies that $J$ is essential in $B$; so $M$ is co-Utumi.

Conversely, assume that $M$ is co-Utumi and let $J$ be a right ideal of $B$ with zero left annihilator, $\mathcal{L}(J)$, in $B$. Then $I_{B} l_{M}(J)=\mathcal{L}(J)=0$ implies, since $M$ is retractable, that $l_{M}(J)=0$, which implies that $J$ is essential in $B$ since $M$ is co-Utumi. Hence $B$ is right Utumi.

EXAMPLES. Any free module, in fact any generator, is retractable, as is any semisimple module, any torsionless module over a semiprime ring and any ${ }_{R} M$ such that $\left(\right.$ Trace $\left._{R} M\right) m \neq 0$ whenever $0 \neq m \in M$.

Examples of Utumi modules are CS-modules, i.e. modules in which every complement submodule is a direct summand (see e.g. [1 and 2] for examples and properties of such modules). To see that a CS-module is Utumi, let ${ }_{R} M$ be any CS-module and let $U$ be any submodule of $M$ such that $r_{B}(U)=0$. Since the essential-closure, $U^{e}$, of $U$ is a direct summand in $M$, there is a submodule, $V$ of $M$ such that $M=$ $U^{e} \oplus V$, and $a b \in B$ such that $U^{e} b=0$ and $v b=v$ for $v \in V$. Then $r_{B}(U)=0$ implies that $b=0$ and so $V=0$ and $U$ is essential in $M$.

If $M$ is a retractable, nonsingular CS-module, then $M$ is also cononsingular, for, by Corollary 3.6 of [3], since $M$ is retractable and Utumi, $B$ is Baer if and only if every essentially-closed submodule of $M$ is a direct summand in $M$. Thus, since $M$ is CS, $B$ is Baer, hence, in particular, right nonsingular, which, by Proposition 1 , implies that $M$ is cononsingular.

Finally, an example of a module which is both Utumi and co-Utumi is obtained when $M$ is taken to be a finite-dimensional (in the sense of Goldie) torsionless module over a ring $R$ which possesses a semisimple two-sided quotient ring, for then $A$ is a semisimple two-sided quotient ring of $B$ [6, Theorems 2.3 and 3.3]. 


\section{REFERENCES}

1. A. W. Chatters and C. R. Hajarnavis, Rings in which every complement right ideal is a direct summand, Quart. J. Math. Oxford Ser. (2) 28 (1977), 61-80.

2. A. W. Chatters and S. M. Khuri, Endomorphism rings of modules over non-singular CS rings, J. London Math. Soc. (2) 21 (1980), 434-444.

3. S. M. Khuri, Baer endomorphism rings and closure operators, Canad. J. Math. 30 (1978), 10701078.

4. $\ldots$, Endomorphism rings of nonsingular modules, Ann. Sci. Math. Québec 4 (1980), 145-152.

5. Y. Utumi, On rings of which any one-sided quotient ring is two-sided, Proc. Amer. Math. Soc. 14 (1963), 141-147.

6. J. M. Zelmanowitz, Endomorphism rings of torsionless modules, J. Algebra 5 (1967), 325-341.

DEPARTMENT OF MATHEMATICS, AMERICAN UNIVERSITy OF BEIRUT, BeirUt, LEBANON 